Volume 9, No.5, September - October 2020

International Journal of Advanced Trends in Computer Science and Engineering

Available Online at http://www.warse.org/IJATCSE/static/pdf/file/ijatcse165952020.pdf

https://doi.org/10.30534/ijatcse/2020/165952020

\title{
Developed Application Software for Wireless Body Area Networks to Reduce Energy Consumption
}

\author{
Seyed Abolfazl Hosseini ${ }^{1}$, Poria Pirozmand ${ }^{2}$ \\ ${ }^{1}$ Faculty of Electrical and Computer Engineering, Aryan Institute of Science \& Technology, Iran, \\ Mr.abolfaz196@yahoo.com \\ ${ }^{2}$ School of Computer and software, Dalian Neusoft University of Information, Dalian 116023, China, \\ Poria@neusoft.edu.cn
}

\begin{abstract}
Measuring vital signs and knowing the patient's condition in diagnosing and treating diseases is so important. The health and medical application can be a good helper for having a healthy lifestyle. The application software that is going to be introduced in this research can be an essential aid for the remote examination of patients. It means that you can send your physical condition and health information to doctors or medical centers with this application software. Thanks to the signals provided by the body's sensors, accurate and permissible reliable physiological estimates can be provided for medical diagnosis on time. There are small sensors that attach to the body and measure vital and environmental signals of the body and send activity signals to the application and help us make our goals for telemedicine come true. Thanks to Smartphone apps, you no longer have to spend a night or a weekend and waiting for doctor's office to be opened. With the help of this application, you can use this emergency technology to reduce power consumption and save time and money. Surveillance software generally has the ability to control patient vital signals that can provide natural and real information for patient recovery. Current efforts are aimed at achieving a system that automatically measures the body's vital signs and presents it to the physician if necessary.
\end{abstract}

Key words : Patient, Physician, Application Software, Reduce Energy Consumption, Wireless Body Area Networks.

\section{INTRODUCTION}

The growth of the sensor network has been widely used in medicine and the healthcare organization. One of these techniques is the use of wireless body networks. Wireless body sensor networks are expected to revolutionize human health monitoring. They are responsible for the comprehensive and cost-effective control of health.

Researchers believe that the Wireless body sensor network systems have led to dramatic changes in the way people view and manage health, such as the way people interact with the
Internet and search for various health-related information. These changes have led to preventive care, which not only improves the quality of life, but also reduces the cost of health care [1].

Online measuring and displaying the patient's vital parameters is an integral part of monitoring patient health with limited resources. One way to collect and send measured data inefficient treatment settings is to use a wireless sensor network.

Current efforts are aimed at achieving a system that automatically measures ominous vital signs and provides them to the physician if necessary; such systems are called patient health monitoring systems. Introducing medical software, the importance of measuring vital signs and knowing the patient's condition in diagnosing and treating diseases is crucial.

For example, what most distinguishes an ICU or CCU section from other sections is careful monitoring of the patient's condition and vital signs. Health and medical applications can both provide useful information to the public and be a good helper for a healthy lifestyle. The application software which is going be introduced in this study is just one example of the hundreds of applications and programs that are widely used today by physicians in other countries. Similar applications have been designed for Iranian physicians they will be further developed in the future, and with the support of the Ministry of Health, rich content support can be created for it.

The new method of remote patient examination can be an essential help to remote areas. Now, instead of going to clinics or hospitals, with this application software, physical condition and health information can be sent to doctors or medical centers [2].

Research is always looking for a solution to meet specific conditions and expectations. Under the same conditions and quality of work, the lower the cost-to-performance ratio, the higher the popularity of the method.

Thanks to the signals provided by the body's sensors, the collected information can be increasingly processed to provide reliable, accurate and permissible physiological assessments in Real-time for medical diagnosis and prescription. Patient monitoring programs generally control 
vital signals and provide objective feedback and information for patient recovery [3].

In such cases, the patient can be kept under the supervision of a physician, in normal physiological conditions without restriction of regular activities and waste costs. It monitors daily activities throughout the daily lives of patients with certain diseases. Hospital monitoring, on the other hand, focuses on cases where patients have to stay in the hospital for intensive care and observation. Sometimes the body's wireless sensor networks can provide continuous measurements of physiological parameters for long periods allowing faster detection of organizational problems and quicker detection of emergencies.

According to the cases mentioned in the above sections, it can be concluded that the treatment of the disease is one of the most important health priorities in the medical community around the world, and that the person must be constantly monitored closely. on the other hand, incomplete and irregular treatment of the disease causes much more problems than failure to treat it. In this way, not only may it cause the death of the person, but it also causes complications that are very resistant to other methods of treatment. For example, one of the most common complications of incomplete and irregular treatment of suscitation is cause of suscitation due to lack of timely use of drugs by the patient.

That's why, The World Health Organization has ordered DOTS treatment to be performed for years in which, in addition to being under the supervision of a physician, the person must be under the supervision of another person and with physical presence and under treatment and care [4].

This requires a lot of money and time for the health systems and the patient, because the patient must have frequent visits to the clinic and hospital staff must waste more time to do this. On days that coincide with official and unofficial holidays, there is practically no monitoring.

Accurate implementation of DOTS is also complicated in remote areas. On the other hand, the use of computer systems and various softwares has provided optimal help in various fields of medicine. Given the above problems and cases, we decided to design an intelligent software that not only helps the doctor and the health system in following up and treating the disease, which is a difficult task and must be done promptly at certain times, but it also acts as an observer for the patient and reminds the person by phone or mobile phone of the time of taking medicine and the time of timely follow-up actions.

The time of the patient's visits for the examination which is taken by the doctor and conducting the necessary tests such as heart tests are also needed to be done in time. The software is also helpful in these cases. The doctor can make sure that the patient uses the medicine correctly, and staff hygiene should also be monitored. Using this software reduces the need for Human resources and saves time. In Figure 1 shows, the architecture of this method is shown in a layered way.

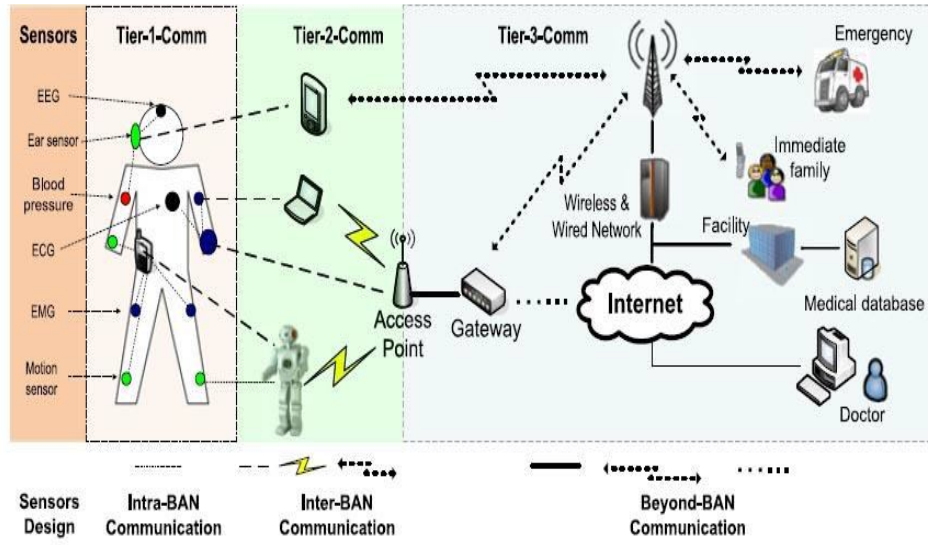

Figure 1: Three-layer architecture of BAN communication system [5]

\section{RELATED WORKS}

The Standard Working Group on the Regional Body Network was launched in December 2007. The working group focused mainly on the medical part of technology application, although some members of the other group also work on the non-medical aspect of technology. Most likely, both medical and non-medical applications from the Bad Regional Network can support the standard network protocol and the physical layer, but due to the requirements of the medical plan, security, quality of service and Mac layer for both programs are different [6],[7]. As a result, the IEEE802 Regional Task Group is developing a physical and Mac standard layer for the regional body network.

The developing standard is expected to fill dificient energy consumption as well as gaps in data rates compared to the power peak diagram [8],[9], Information and communication technology has succeeded in improving health care and enabling people to manage their health [10],[11].

Some technologies show a limited impact on health compared to others that have a direct effect on improving health care for individual users, such as electronic health records [12] telemedicine [13], consumer health information [14].

Decision-making applications are often used to provide feedback on issues such as insulin levels, food intake, and physical activity. In this study, only a few articles described the decision support form [15],[16] and an application dedicated to decision-making that is usually stored on patients' devices. The app can calculate data and provide suggestions for health-related actions based on user activity with the app. In some cases, such programs even link to electronic health records, which allow the physician to review the data and provide feedback.

An application such as Preuveneers [15] provides information on physical activity and blood sugar levels, and a program to calculate the appropriate insulin dose for the patient. However, most of the programs reviewed only offer insulin dose recommendations, such as the RapidlCalc Diabetes manager, which covers more aspects of patients' physical lifestyles [17]. Bass Vanmeulebrouk et al. showed that open Source-based GIS software could be used to provide useful 
information in both the treatment and planning of diseases [18].

Alireza Saleki, with the help of Kermanshah University of Medical Sciences, developed software for the use of maternal mortality, which has won various awards in the country and the province and is an essential step in dealing with pregnant mothers [19]. The TB information management system software, which is used only in the United States, Colombia and some parts of the Caribbean, is related to the reporting and analysis system and differs from the programs of the World Health Organization and our country [20].

Robert McMaho et al. showed that the use of health management technology based on health information technology is a critical aid in the treatment, follow-up and monitoring of patients. in addition to providing valuable information, it is also cost-effective. [21]. A review study in 2010 showed that the use of information technology (IT) in drug integration is a necessary process and is used to make the treatments useful and safe in the patient [22].

A 2009 study hoped that the use of health information technology in the United States would attract the attention of politicians, which would help treat patients and bring economic benefits [23]. There is currently very little research on what patients need to know about E-health, especially those with chronic diseases, and what they may need to use. In order to understand the level of knowledge and interest of patients to see how they use E-health programs to support themselves.

A 2013 study hoped that the use monitoring system that has the capability to monitor physiological parameters from multiple patient bodies[24].

A model of Tele ECG is supported with a Bluetooth subsystem which would transfer the obtained ECG output to a cellular device and can be easily sent to expert for medical advice using the MMS service[25].

\section{SUGGESTED APPLICATION}

A new system that allows physicians to diagnose patients' conditions remotely, and whenever and wherever people can monitor their vital signs, is called HealthCare, which is compatible with mobile phones and tablets (Figure 2) so that people can have permanent and special care. Visualization of HealthCare will improve the delivery of healthcare and patient care; It means that the doctor from anywhere in the world can receive the patient's condition and vital signs transmitted by through this system and of course the wireless body area's sensors, And prescribe the required medications based on the results of your tests and diagnosis.

HealthCare is often used for patient health check-ups, chronic disease management, medication management, specialist counselling and some other clinical services that can be provided remotely. This application allows you to have doctors diagnose your disease and prescribe medication if necessary. Your doctor can diagnose your health during an online appointment and even prescribe medication if necessary. Provide your medical data to your doctor and get advice remotely. You can safely and quickly share your ultrasound images, tests, and other information with your doctor. With this type of software, patients no longer need to wait for hours in doctors' offices to visit and spend money to travel to these centers.
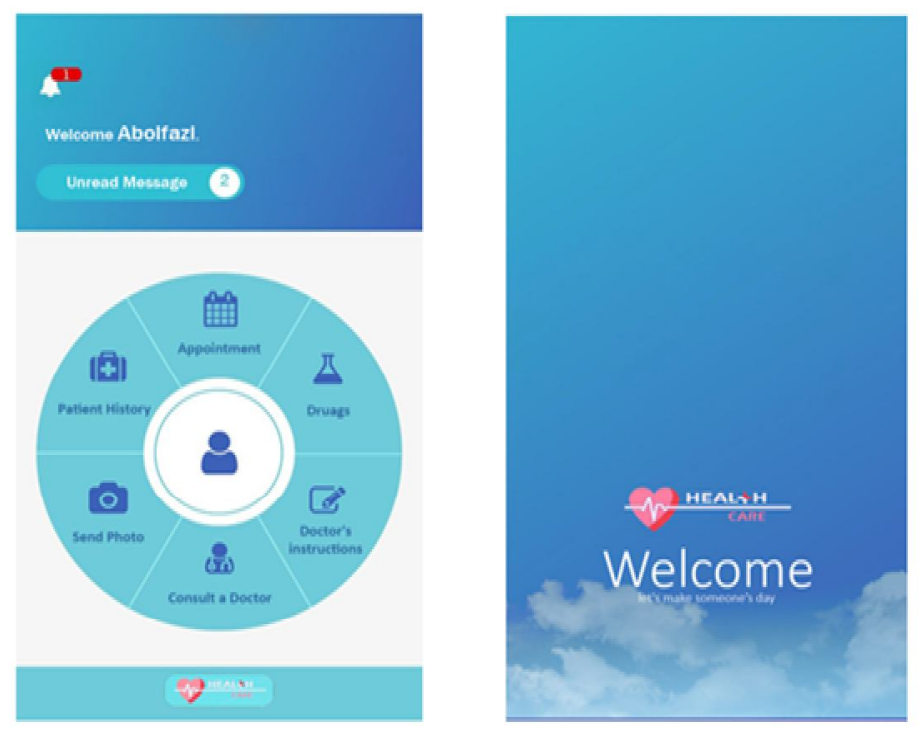

Figure 2: The HealthCare application

There are small sensors that attach to the body and measure vital and environmental signals of the body and send activity signals to the application and help us make our goals for telemedicine come true. Thanks to Smartphone apps, you no longer have to spend a night or a weekend and waiting for doctor's office to be opened. With the help of this application, you can use this emergency technology to save time and money.

Today, applications are a source of revenue for various businesses. Investing in an attractive and quality medical care app is not comparable to the income it will generate for designers and investors. Global demand for care programs is expected to generate $\$ 2,000$ billion in revenue by 2023 , in a way that it will be increased annually with a compound growth rate of $3.41 \%$ from 2013 to 2023.

Global urgent care app market, by region, by value, 2013-2023

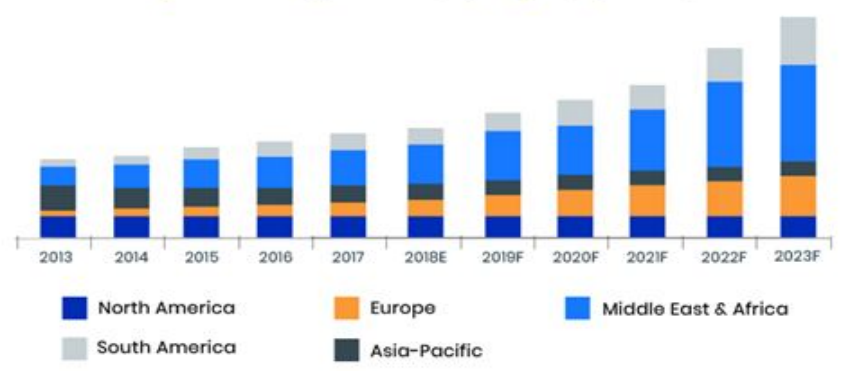

Figure 3: Chart of market situation and the future of medical care application 
Figure 3 shows that, The main reason for the growth of the market for remote care applications is the growing use of smartphones and the significant benefits of such applications.

\section{THE PROPOSED METHOD}

Network reliability directly affects the quality of patient monitoring and, in the worst case, may lead to death due to failure to diagnose an event that threatens the patient's life. However, due to bandwidth and energy limitations, techniques used to build reliability in older networks, such as the packet retransmission mechanism in the TCP protocol, is not very practical for WBAN networks.

Researchers have proposed several ways to improve reliability. Power management in physical networks is a significant practical issue. Power consumption can be minimized by documenting the physical layer and MAC processes.

A physical layer with the right coding and modulation selection can increase the likelihood of sending a packet successfully. The higher the probability, the more energy is saved. The power consumption of a node can be optimized by increasing the likelihood of successful packet sending. On the other hand, the MAC layer can have a vital role in consumption by using techniques such as channel access techniques, intelligent signalling techniques and also the use of optimal closed structure [26].

\subsection{Proposed adaptive MAC protocol in wireless network physical}

PURPOSE-MAC is based upon TDMA approach to minimize energy consumption. PURPOSE-MAC assigns Guaranteed Times Slot (GTS) to each sensor node for communication based upon the requirements of sensor node. We assume a star topology; a Central Node $(\mathrm{CN})$ collects data from sensor nodes and communicates with a Monitoring Station (MS), direct or through an Access Point (AP). We assume $\mathrm{CN}$ with single transceiver where $\mathrm{T}^{\text {Frame }}$ is divided into three parts: Contention Free Period (CFP) for communication with sensors, Contention Access Period (CAP) to accommodate emergency or on-demand traffic and time TMS for communicating sensor nodes' data to MS.

\subsubsection{Time Slots Assignment}

PURPOSE-MAC uses an adaptive scheme for Time Slot (TS) and Guard Band Time(GBT). The selection of free RF channel is followed by Time Slot Request (TSR) packet; transmitted by nodes to $\mathrm{CN}$ which includes information about the node's Time Slot (TS) for communication as well the data rate. Assignment of variable TSs and $T^{\mathrm{GB}}$ is followed by Time Slot Request Reply (TSRR) from CN to nodes. To avoid interference between two successive time slots $T^{\mathrm{GB}}$ is inserted. We calculate $T^{\mathrm{GB}}$ as follows:

$$
\begin{aligned}
& T_{n+n+1}^{G E}=\frac{G_{F}}{100} \times \frac{1}{2}\left(T S_{n}+T S_{n+1}\right) \\
& T_{1}^{G B}=\frac{G_{F} \times T S_{1}}{100} \quad, \quad T_{n}^{G E}=\frac{G_{F} \times T S_{n}}{100}
\end{aligned},
$$

where $\mathrm{G}^{\mathrm{F}}$ is the guard band factor which depends upon the average drift value, $\mathrm{T}_{1}{ }^{\mathrm{GB}}$ is inserted before first time slot, and $\mathrm{T}_{\mathrm{n}} \mathrm{GB}^{\mathrm{B}}$ is placed before $n$th time slot. This mechanism provides, almost collision-free and reliable TSs with reduction in Gunergycensumption. $\mathrm{T}^{\mathrm{GB}}$ assignment is shown in Fingure 4 ith MS

Contention access period

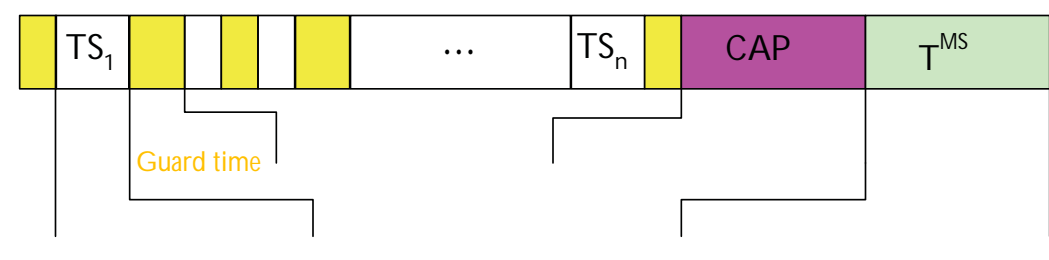

Figure 4: Time slots assignment with guard-band time.

\subsubsection{Synchronization Mechanism}

TDMA schemes require extra energy cost for periodic synchronization [27]. Proposed MAC uses a novel synchronization mechanism to avoid collision and energy consumption. In order to communicate efficiently within the assigned TSs, CN needs synchronization with nodes. Within expected TS, CN listens for data packet. Upon the reception of data packet, $\mathrm{CN}$ compares expected reception time with current reception time.

\subsection{Energy consumption}

In this research, we assume the energy consumption of sensor and processing units is constant. We consider the intermittent traffic pattern; for example, sensor nodes send periodic information to the central node at specified intervals. Most time sensor nodes remain dormant. They stay awake at regular intervals to send data. the following equation to measure energy consumption.

$E_{\text {Total }}=\sum_{k=1}^{N} E_{\text {sleep }_{k}}+\sum_{k=1}^{N} E_{\text {Active }_{k}}$

Energy consumption is a function of the current time and design of the voltage source for a particular job. They still consume energy when the nodes go to sleep.

$$
\begin{aligned}
& T_{\text {Sleep }}=T_{\text {Frame }}-T_{\text {Active }} \\
& E_{\text {Total }}=T_{\text {Sleep }} \times I_{\text {Sleep }} \times V
\end{aligned}
$$


$I_{\text {Sleep }}$ is the current design of the voltage source during sleep. In $T_{\text {Active }}$ nodes receive and send information and wait for confirmation. In the change from sleep to active state and from active to sleep state, energy is consumed. The energy consumed for all of these tasks is counted as $T_{\text {Active }}$ energy.

$E_{\text {Active }}=2 \times E_{S W}+E_{\text {Trans }}+E_{\text {Rec }}+E_{\text {Tout }}$

In this formula $E_{S w}$ is a change of state energy, $E_{\text {Trans }}$ is the energy of transmission, $E_{R e c}$ is the energy received, and $E_{T O u t}$ is stopping energy. We describe these terms in detail in the following small sections.

4.2.1. Change energy. Frequent transmitter-receiver switching between two modes leads to high energy consumption. The following equation determined The energy used to change the transmitter-receiver:

$E_{\text {SW }}=T_{\text {Switch }} \times I_{\text {Switch }} \times V$

In this equation, $T_{S w i t c} \square$ is required to change the transmitter-receiver between sleeping and waking state, and $I_{\text {Switc } \square}$ current is needed.

4.2.2. Transmission energy. The equation below relates the transmission energy to the packet length $\mathrm{P}$, the time required to send a single bit of $T_{B y t_{\mathrm{e}}}$, the flow diagram during transmission of $I_{\text {Trans }}$, and the voltage source of $\mathrm{V}$.

$E_{\text {trans }}=P \times T_{\text {Byte }} \times I_{\text {Trans }} \times V$

4.2.3. Receiving energy. Receiving energy is the energy used to receive packets and process related to them. The energy received is expressed as follows:

$E_{\text {Rec }}=P \times T_{\text {Byte }} \times I_{\text {Rec }} \times V$

In this formula, $I_{R e c}$ is reception during progress, $T_{B y t_{\mathrm{e}}}$ is the allowed bit time, $\mathrm{P}$ is the length of the packet, and $\mathrm{V}$ is the voltage source.

4.2.4. Stop energy. It is the energy that is consumed after the transfer, and before receiving the ACK packet, for current from $I_{\text {TOut }}$, we use $T_{\text {TOut }}$ for time. we use $\mathrm{V}$ for the voltage source to calculate the energy consumption during the stop time in the following equation.

$$
l_{T} \text { out }=I_{\text {Tout }} \times I_{\text {Tout }} \times V
$$

\section{RESEARCH RESULTS}

We use MATLAB software to measure and compare energy efficiency. Comparison of energy consumption, the transmitter-receiver energy consumption of radiofrequency was investigated. We use the crossover information plate pattern (MICAz) for energy consumption, which is shown in Table 1. Packages are randomly placed with an average package error probability of 1 to $20 \%$. The time frame size used in the simulation is TFrame $=30$ seconds. The simulation was used for 3 sensor nodes, and we used to calculate the energy consumed for $\mathrm{N}=100$. Figure 6 shows a comparison of the proposed MAC energy with IEEE802.15.4 and AR-MAC are showing that the power consumption of IEEE802.15.4 increases with increasing probability of closed error. This increase in power consumption is due to the need for higher energy consumption CSMA/CA performance in IEEE802.15.4 . AR-MAC sets guaranteed time intervals for sensor nodes to communicate, overcome collision, and eavesdrop. The reason for choosing IEEE802.15.4 and AR-MAC algorithms and comparing them with the proposed algorithm can be considered as the basis of the protocol design method with TDMA method. So we have tried to compare the proposed algorithm with other valid and new algorithms in a entirely fair situation. The most crucial difference between the proposed pattern algorithms is the attempt to minimize the number of access channels and the use of a unique routing method. Table 1 compares the proposed algorithm and other algorithms in terms of conditions and other similar and distinct features.

MAC-proposal offers better performance than the other two approaches. Our PURPOSE - MAC minimizes more power consumption than the previous two protocols by trying to reduce the number of access channels, adaptation, bandwidth allocation, and GTSs for communication, MAC-suggestion for communication. To analyze longevity and throughput, we assume that each node is initially supplied with $0.5 \mathrm{~g}$ of energy.

The proposed MAC indicates better network lifetime performance than the peer-to-peer protocols given in the network, As shown in Figure 5. From the simulation results, we conclude that all the features mentioned throughout this article enable a Mac to improve peer protocols.

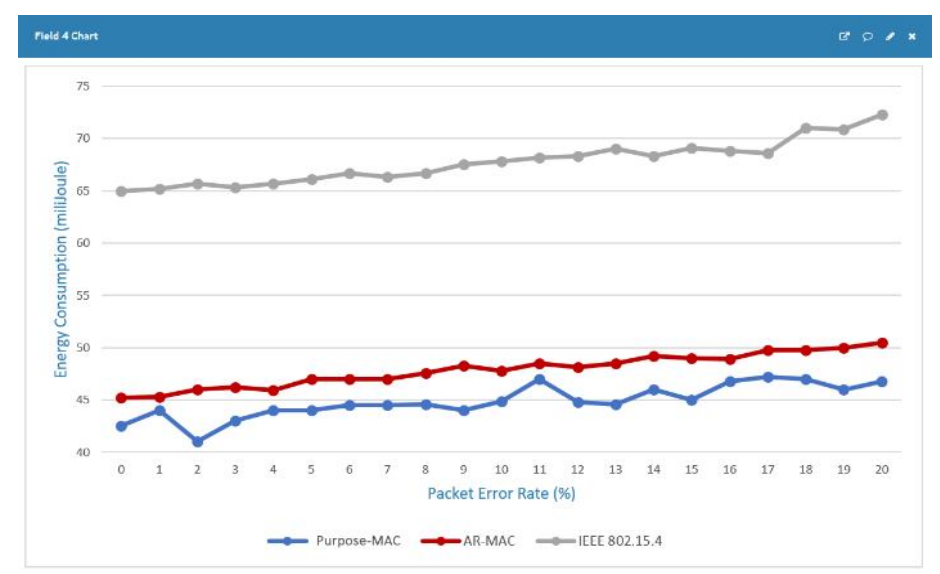

Figure 5: Energy consumption analysis for 100 cycles 
Table 1: Simulation Parameters Valu

\begin{tabular}{ll}
\hline Parameter & Value \\
\hline \hline Voltage & $3.7 \mathrm{v}$ \\
$\mathrm{T}$ frame & $30 \mathrm{~s}$ \\
Current & $20 \mathrm{~mA}$ \\
Transfer current & $200 \mathrm{~mA}$ \\
Receive current & $180 \mathrm{ma}$ \\
Active radio current & $50 \mathrm{ma}$ \\
Inactive (idle listening) & \\
Byte transmission time & $16 \mathrm{us}$ \\
Package length per byte & $\mathrm{P}=12 \mathrm{byte}$ \\
ACK Packet length per byte & $\mathrm{Ack}=2$ \\
Number of sensor nodes & 3 \\
Number of cycles & $\mathrm{N}=100$ \\
\hline
\end{tabular}

\section{CONCLUSION}

Given the importance of smart health services for the elderly, the sick and children, our solution to improve health and provide remote social care is to consider the quality of service. As you know, the concept of quality is inherently a human concept. If a person is satisfied with something or that thing meets his expectations, then it can be said that he has an acceptable quality by that person's standards. If a person is dissatisfied and his expectations are not met, then the quality will not be fair to him. On the other hand, if the product, if possible, exceeds a person's expectations, then it will have a deficient quality in terms of the person's idea. Because quality is a fundamental human concept, it is tough to consider and guarantee the quality that should be created in computing systems and applied to the applications that run on them. Using this application will not replace face-to-face counselling, but will provide an alternative way to communicate between physicians and patients. Hence, It can improve the efficiency of medical processes.

With the HealthCare program, anyone can quickly access their doctor's advices and prescriptions when they need medical care. Therefore, by completing, developing and designing the optimal sample of HealthCare program based on wireless sensors, we can focus on fast and quality care of patients.

\section{REFERENCES}

1. C. Otto, A. Milenkovic, C. Sanders \& E. Jovanov. System architecture of a wireless body area sensor network for ubiquitous health monitoring, Journal of mobile multimedia, 1(4), pp. 307-326, Oct 2006.

2. E. Aguirre. Design and performance analysis of wireless body area networks in complex indoor E-Health hospital environments for patient remote monitoring, International Journal of Distributed Sensor Networks, 12(9), pp. 15-32, 2016.

3. A. Hadjidj, M. Souil, A. Bouabdallah, Y. Challal, H. Owen. Wireless sensor networks for rehabilitation applications: Challenges and opportunities, Journal of Network and Computer Applications, 36(1), pp. 1-15, 2013.

4. M. Naseh, L. Mirhaghani. National Guide to Fighting Tuberculosis. Second Edition, Tehran, Ministry of Health, Treatment and Medical Education, 2010.

5. K. Sohraby, D. Minoli, \& T. Znati. Wireless Sensor Networks: Technology, Protocols and Applications, Published by wiley interscience, 2017.

6. J. Bassi , F. Lau, S. Bardal, Use of Information Technology in Medication Reconciliation: A Scoping Review, Ann Pharmacother, 44(5), pp. 1274-1281, May 2010.

7. E. Rashedi, H. Nezamabadi-pour, S. Saryazdi. BGSA: Binary Gravitational Search Algorithm, Journal of Information of Technology, 42(1),pp. 2-8, Sep 2015.

8. J. Rabaey. PicoRadio Supports Ad hoc Ultra-low Power Wireless Networking, IEEE Trans. on Computer, vol. 33, pp. 42-48, July 2003.

9. S. Meyer and A. Rakotonirainy. A Survey of Researchon Context-Aware Homes, Proceedings of the Australasian information security workshop conference on ACSW frontiers, vol.21,pp. 159-168, Jan 2003.

10. R. Katz, J. Kahn, \& K. Pister, Mobile Networking for Smart Dust, in Proceedings of the 5th Annual ACM/IEEE International Conference on Mobile Computing and Networking (MobiCom'99), Seattle, pp. 271-278, Aug 2014.

11. L. Jain, K. Singh. Application of IT in Biomedical Field: Present Status and Future Prospects, International Journal of Scientific \& Engineering Research, Vol 3, Issue 2, Feb 2012.

12. M. P. Gagnon, F. Légaré, M. Labrecque, P. Frémont, P. Pluye, J. Gagnon, J. Car, C. Pagliari, M. Desmartis, L. Turcot, K. Gravel, Interventions for promoting information and communication technologies adoption in healthcare professionals, Cochrane Database of Systematic Reviews, doi:10.1002/14651858, 2009.

13. N. Rowe, S. Gibson, S. Morley, E. A Krupinski, Ten-year experience of a private nonprofit telepsychiatry service, Telemedicine \& E-Health:the official journal of the American Telemedicine Association, 14(10), pp. 1078-1086, Dec 2008.

14. J.C. Crosson, C. Stroebel, J. G. Scott, B. Stello, B. Crabtree, Implementing an electronic medical record in a family medicine practice: communication, decision making, and conflict, Annals of Family Medicine, 3(4), pp. 307-311, Jul 2005.

15. D. Preuveneers, \& Y. Berbers, Mobile phones assisting with health selfcare: a diabetes case study, Proceedings of the 10th International Conference on Human Computer Interaction with Mobile Devices and Services, Amsterdam, The Netherlands, pp. 177-186, Sep 2008. 
16. C. C. Quinn, M. D. Shardell, M. L. Terrin, E. A. Barr, S. H. Ballew, A. L. Gruber-Baldini, Cluster-randomized trial of a mobile phone personalized behavioral intervention for blood glucose control, Diabetes Care, 34(9), pp. 1934-1942, Sep 2011.

17. G. Enterprises., RapidCalc Diabetes Manager, 2016.

18. B. Vanmeulebrouk, U. Rivett, A. Ricketts, M. Loudon, Open source GIS for HIV/AIDS management. International, International Journal of Health Geographics, 7(1), pp. 1-16, Dec 2008.

19. A.R. Saleki, SH. Khosravi, F. Foroghi, R. Qasemi,A. Rashidi, Q. Nasiri., F. Government, Design and build smart software Hello pregnant mother at risk, Moleh Kermanshah Medical Sciences, Code.1392,3066 articles, April 2013

20. Tuberculosis Information Management System (TIMS)" http://www.cdc.gov/tb/programs/tims/tims.htm , 2008.

21. R. McMahan, D. Pharm ‘M. MATS, Operationalizing MTM Through the Use of Health Information Technology , Supplement to Journal of Managed Care Pharmacy JMCP, 14(2),pp. 18-21,Mar 2008.

22. J. Bassi ,F. Lau , S. Bardal, Use of Information Technology in Medication Reconciliation: A Scoping Review, Ann Pharmacothe ,pp. 52-68, May 2010.

23. DC. Balfour, S. Evans, J. Januska, HY. Lee, SJ. Lewis, SR. Nolan, M. Noga,C. Stemple, K. Thapar, Health information technology-results from a roundtable discussion. Journal of managed care pharmacy, Jan 2009, 15(1 Supp A),pp. 7-10.

24. M. Aminian, HR. Naji, A hospital healthcare monitoring system using wireless sensor networks, International Journal of Scientific \& Engineering Research, 4(02) ,pp. 121, Feb 2013.

25. D. Hazra, V Manasa, Application of Biosensor in Tele ECG using Wireless Technology, International Journal of Scientific \& Engineering Research, 4(3),pp. 1-7, Mar 2013.

26. J. Hill ,R. Szewczyk ,A. Woo ,S. Hollar ,D. Culler ,K. Pister, System Architecture Directions for Networked Sensors ,Proceedings of the 9th International Conference on Architectural Support for Programming Languages and Operating Systems, vol. 28 , pp. 93-104, Nov 2013.

27. Q. Zhou, X. Cao, S. Chen, and G. Lin, A solution to error and loss in wireless network transfer, in Proceedings of the International Conference on Wireless Networks and Information Systems (WNIS '09), pp. 312-315, December 2009. 